\title{
Assessment and mitigation of voltage violations by solar panels in a residential distribution grid
}

\author{
Tom Verschueren*, Kevin Mets*, Bart Meersman ${ }^{\dagger}$, Matthias Strobbe*, Chris Develder*, and Lieven Vandevelde ${ }^{\dagger}$ \\ * Dept. Information Technology - IBCN, Ghent University - IBBT, Ghent, Belgium \\ $\dagger$ Dept. Electrical Energy, Systems and Automation (EESA) - EELAB, Ghent University, Ghent, Belgium \\ Email: chris.develder@intec.ugent.be, bart.meersman@ugent.be
}

\begin{abstract}
Distributed renewable electricity generators, such as solar cells and wind turbines introduce bidirectional energy flows in the low-voltage power grid, possibly causing voltage violations and grid instabilities. The current solution to this problem comprises automatically switching off some of the local generators, resulting in a loss of green energy. In this paper we study the impact of different solar panel penetration levels in an residential area and the corresponding effects on the distribution feeder line. To mitigate these problems, we assess how effective it is to locally store excess energy in batteries. A case study on a residential feeder serving 63 houses shows that if $80 \%$ of them have photo-voltaic (PV) panels, $45 \%$ of them would be switched off, resulting in $482 \mathrm{kWh}$ of PV-generated energy being lost. We show that providing a $9 \mathrm{kWh}$ battery at each house can mitigate some voltage violations, and therefor allowing for more renewable energy to be used.
\end{abstract}

\section{INTRODUCTION}

The power grid is moving away from the current centralized power generation paradigm. With governments promoting local renewable power generation at residential sites, distributed power generation is gaining in popularity. Environmental concerns and efforts to become less dependent on fossil fuels are the driving force for the replacement of traditional energy sources by green alternatives. The EU 20-20-20 targets aim for a reduction in $\mathrm{EU}$ greenhouse gas emissions of at least $20 \%, 20 \%$ of EU energy consumption to come from renewable energy sources, and a reduction in energy consumption of $20 \%$ [1]. Currently, a lot of wind farms are constructed, offshore as well as on land, and in residential areas more and more houses are covered with solar panels.

By combining power grid technologies with information and communication technologies a future smart grid will be able to deal with the unpredictable and distributed nature of these new forms of power generation. Typical issues of the future power grid stemming from the presence of distributed generation (DG) include [2]: voltage and frequency instabilities as a result of local power generation, power security issues resulting from bidirectional energy flows. In addition, given the less predictable nature of renewable energy sources, the demandsupply matching problem becomes more challenging, and associated control algorithms that cater for flexibility of loads (i.e., that can be shifted in time) become more complex.

In residential neighbourhoods with houses equipped with photo-voltaic (PV) panels, there is often a mismatch between the production and consumption of the power generated by these solar panels, since maximum production is often reached at moments when the inhabitants don't need a lot of power. For example because they are working during daytime or they are on holiday during the summer. When they arrive at home and start cooking, washing, etc. solar production might be low, especially during the winter. Unfortunately, electricity has to be used the moment it is generated as the power grid represents the ultimate just-in-time product delivery [3].

When PV power production is high and consumption is low, extra power is injected into the grid, possibly causing voltage violations and making the grid unstable. On the other hand, to ensure that all households can be served in case consumption is high and production is low, the presence of PV panels does not necessarily decrease the required amount of (spare) capacity that must be available all the time, requiring high investments and resulting in expensive energy bills.

In this paper we study the impact of distributed solar power production in a distribution grid by determining power flow and voltage fluctuations at the grid connection of each household, taking available generation capabilities (in casu, PV panels) and consumption patterns into account. Thus, we will assess which PV panels will be turned off because of voltage violations, and as such assure grid stability. This has the undesirable side effect that some of the PV energy is actually wasted, and not injected into the grid. We subsequently assess how we can alleviate these problems by providing local storage capacity with batteries.

The remainder of this paper is structured as follows. In Section II related work is presented. The simulation tool we developed and used to obtain our case study results is briefly described in Section III. The set-up of our case study, including the models for generation, consumption and storage, is detailed in Section IV. The results and discussion of the observerd effects of distributed PV generation in a residential area is presented in Section V. The final Section VI summarizes our conclusions and provides some thoughts on future work.

\section{RELATED WORK}

With the ever increasing instalment of renewable energy sources at residential areas, the impact of distributed generation on electrical power systems has been of major interest [4][6].

The effect of the capacity and location of distributed generators in the distribution grid has been discussed in [6]. 
That analysis is based on the voltage stability at each of the 69 nodes in the considered distribution system, which is calculated using the steady state voltage index method [7].

Different approaches at determining system stability with different penetration levels of DG are used in [5], which, apart from the the voltage stability metrics, includes stability analysis and the assessment of the oscillatory behaviour of the power system. The power system consists of a high voltage and two low voltage networks, with a total number of 56 enduser nodes, each connected to a fuel cell and micro turbine.

Battery energy storage systems are interesting to support the stability and reliability of the power grid and for efficiently using the energy generated by unpredictable and intermittent sources such as solar panels and wind turbines. Especially electrical vehicles receive a lot of attention as possible power storage and source as they have a higher economic potential than dedicated storage systems (V2G principle [8], [9]).

The authors of [10] present recent advances in energy storage systems with respect to ease of maintenance, costs and size. Different types of energy storage systems are discussed including different types of batteries, flywheels, superconducting magnetic energy storage, ultracapacitors, microturbines and fuel cells. Also [11] gives an overview of different kinds of energy storage systems together with a number of benefits, both technical (e.g. grid voltage support, peak shaving, reliability, etc.) as financial (e.g. reduction of need for extra generation capacity, increased revenue from renewable sources, etc.). Furthermore, some modelling and simulation approaches are discussed as well as a number of challenges that need further research.

In [12], the siting and sizing of electricity storage is optimized stochastically using an multi-objective genetic algorithm. The optimal integration of battery energy storage units is analysed with respect to several objectives including minimization of total installed power and energy capacity, minimization of large voltage deviation probabilities and maximization of total revenue on a power exchange market.

The work of [13] presents a game-theoretic framework for modelling storage devices in large-scale systems where each storage device is owned by a self-interested party that aims to maximise its monetary profits. The designed storage strategy results in reduced peak demands and lower electricity bills.

Besides already mentioned benefits such as reducing peak demands, avoiding voltage violations, lowering grid investment costs and reducing energy bills for the end users, battery energy storage systems have the additional benefit of being able to protect the privacy of the end user by hiding information contained in consumption data [14].

Our work discussed below also addresses issues of storage in residential scenarios, but differs from the above works in that it assesses how storage can be exploited to counter voltage violation issues stemming from fairly high penetration of residential photo-voltaic installations.

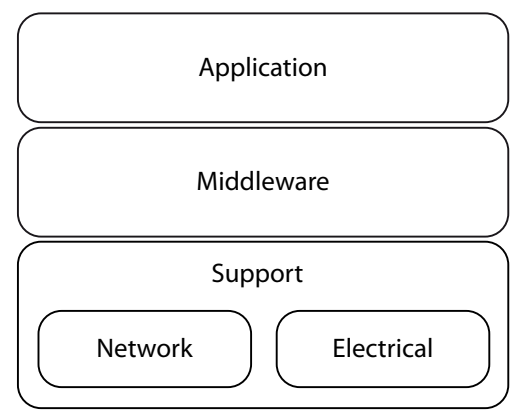

Fig. 1. Three-layer architecture of the simulation environment.

\section{SimUlation FRAMEWORK}

To study smart grid scenarios such as the one we are addressing in the current paper, we resort to simulations. To mitigate the supply and demand challenges stemming from e.g. the intermittent nature of renewable sources, it is clear that information and communication technologies (ICT) can play a major role and will be crucial to enable smart grid applications [15], [16]. Whereas both the communication network and the power system research communities have developed ample simulation tools, we believe there is a need for an innovative framework that couples both worlds in an integrated view. Therefore, we have developed a modular simulation framework based on OMNeT++ [17], reusing some of the communication network modules already available, but adding extra models for the electrical network. Below, we give a high level overview of the simulator design, and outline the power model used. For details, we refer to [18].

\section{A. High-level design}

As sketched in Fig. 1, we designed our smart grid simulation environment as a layered architecture, comprising three major layers: application, middleware and support layers. The application layer consists of high-level applications or services, for example advanced meter reading services, demand side management services, or billing services. The services in the application layer make use of the middleware layer, which provides generic functionality that can be used by any service. This includes e.g. service/device discovery functions as well as a message-based communication interface to send messages between service components, independent of underlying networking technology (e.g. ZigBee or PLC; TCP or UDP). The goal of this middleware layer is to support a broad range of applications while reducing the effort required to develop these services to a minimum. The support layer, composed of the network and electrical components, provides support functions for the layers above. Communication between services is simulated by the network component that provides simulation models for multiple types of physical media and communication protocols. The simulation environment must be able to model and interact with (virtual) electrical devices. This is supported by the electrical component of the simulator, as outlined below. Basic electrical models are provided (e.g. 
PV panel, battery, electric vehicle), but the user can add his own models. The characteristics of the specific models used in this paper are discussed in Section IV.

\section{B. Power network model}

Our current research interest mainly pertains to the distribution grid, since the major research questions to be addressed are in this part of the power network. Therefore, we implemented a distribution grid model to allow assessing potential problems, including in particular voltage violations, stemming from distributed generation. Our current model applies to radial feeder networks, comprising one or more feeders that in turn may include other subfeeders. Loads or generators can be connected anywhere along the feeders. The model is based on the fast harmonic simulation method presented in [19]. It uses an iterative forward/backward sweep method to analyse the radial distribution system. Each iteration consists of two parts: the backward and forward sweep. The backward sweep determines the currents in every node, based on known voltages in each node. Subsequently, the currents in all network branches are determined. Next, the forward sweep determines the voltage at every node. After each iteration, the voltage at every node is compared with the voltage in the previous iteration. If the difference is below a certain threshold, the iteration process is stopped. We realized the iterative method as a MATLAB module, which is linked to the $\mathrm{OMNeT}++$ modules through appropriate interfaces, to allow e.g. specification of the number of houses, the loads etc.

\section{CASE STUdy SET-UP}

To assess the impact of PV panels on a distribution feeder, we have considered a radial feeder topology, as presented in [19], comprising three feeders, with 21 houses on each of them. All of the houses are connected on the same phase, and are placed equidistantly on feeders with a length of 1000 meters. The low voltage lines used in this simulation are underground cable lines.

To assess the impact of the PV panels, we will compare three cases:

- NoPV: will illustrate the baseline scenario without any PV panels or batteries, as a reference case.

- PV-ideal: will show the idealized case where we do not take into account any voltage violations when assessing energy flows. Thus, in this case all produced PV energy can always be used (either locally in the house, or pushed into the grid).

- PV-real: will show the realistic case, where PV panels are shut off the grid when they cause voltage problems. Therefore, in this case we will have no voltage violations, but part of the generated PV energy will be lost.

- PV+batt: will indicate how the use of batteries may mitigate the voltage violations. In this case, we will assume each household with a PV installation is also equipped with a battery. This battery will then be used during daytime to store excess energy produced by PV panels, and to release it in the evening/night time.

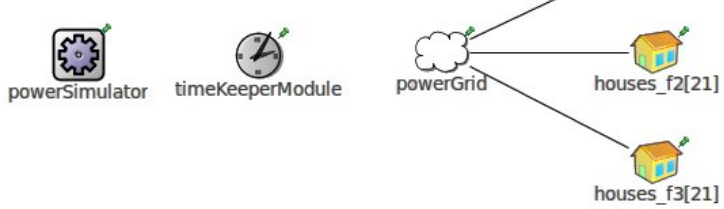

(a) Overall set-up

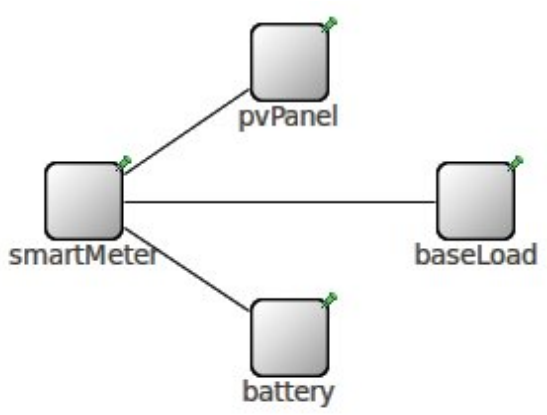

(b) House module

Fig. 2. The case study set-up in the simulator.

The overall layout of our case studies, as represented in our simulation framework is illustrated in Fig. 2. The main setup of the OMNeT++ simulation environment is shown in Fig. 2(a), where each house icon on the right represents multiple (21) House modules. All of those house modules are connected to a specific feeder, coming from the powerGrid module. The powerGrid module provides a high level representation of a distribution grid, and allows for the configuration of several feeders that define the distribution grid.

A House module, detailed in Fig. 2(b), acts as a container for a number of devices that influence the electrical behavior of the house. The baseload models the consumption of electricity by the whole of all household appliances. All these devices, as well as the PV panel and the battery, are connected to the smart meter of the house which keeps track of the net power supplied to (or possibly by) the home. Thus, in our case studies, each household will be characterised by

- A base load profile: the local electricity consumption profile stemming from typical household appliances;

- A PV panel: the solar panel, if present;

- A battery, if present.

The properties of these models used are described in more detail in the subsections below.

\section{A. Base load profile}

The base load profiles are based on the synthetic load profiles (SLP), made available by the Flemish Regulation Entity for the Electricity and Gas market (VREG) [20]. These SLP profiles model typical user consumption using statistical averages on real life data, as measured by the VREG: an SLP curve contains for each 15 minute interval, over a complete year, the amount of energy consumed (in $\mathrm{kWh}$ ). We used such 
SLP data representing a residential consumers. In particular, we used data for the first of July of the 2011. profile published by VREG. The total yearly power consumption was set at $3500 \mathrm{kWh}$, corresponding with normal residential usage [20].

\section{B. PV panel model}

To model the production of the PV panels, the same approach as for the base load profiles has been used. The European PV GIS [21] database contains averaged solar power irradiation profiles, similar to the (consumption) SLP files. The the same generation profile (average solar power irradiance in July at Ghent, Belgium) is used for all PV panels in the distribution network, albeit altered with a random uncertainty factor of 5 percent a each location. Next to this irradiance profile, the PV model also takes a surface (squared meters) as parameter. By multiplying the irradiance value $\left(\mathrm{W} / \mathrm{m}^{2}\right)$ with the surface $\left(\mathrm{m}^{2}\right)$, we get a simplified model omitting any efficiency factors. The results presented in the next section used an average PV panel surface of $6 \mathrm{~m}^{2}$. To account for variances between households, we randomly assigned PV surfaces and efficiency factors drawn from a uniform distribution with aforementioned averages and a maximal deviation of $\pm 10 \%$.

\section{Battery model}

We used a simplified, lossless model of battery cells, which we assume to be characterized by the following parameters: (i) maximum storage capacity $E_{B}$ (in $\mathrm{kWh}$ ), (ii) maximum decharge power $P_{\text {out }}$ (in $\mathrm{kW}$ ), and (iii) maximum charge power $P_{\text {in }}$ (in $\mathrm{kW}$ ). Thus, when charging a battery at time $t$, it will draw $p(t) \leq P_{\text {in }}$ from the grid, while it will discharge at $p(t) \leq P_{\text {out }}$. For simplicity, in this work we disregard any battery inefficiencies such as self-discharge, loss of capacity (e.g. due to memory effects), etc. For the case study below, we assumed $P_{i n}=1 \mathrm{~kW}$ and $P_{\text {out }}=0.7 \mathrm{~kW}$.

\section{RESULTS AND DISCUSSION}

Figure 3 below show the power levels (W) as observed at the main feeder of the distribution grid, and hence showing the total net power consumption or production of the entire distribution grid network. Three cases are presented, for different PV penetration levels: we consider $50 \%, 70 \%$ or $80 \%$ of the houses to have a PV installation..

Figure 3 shows both the PV-ideal and PV-real scenario. In the $50 \%$ penetration case, no voltage violations occurred and therefor no PV panels were disconnected from the grid, resulting in a match between the PV-ideal and PV-real measurements (note that therefor the curves in Fig. 3(a) overlap). For 70\% or $80 \%$ penetration however, the injected energy by the PV panels reaches a sufficiently high threshold which causes overvoltage problems in the grid. When a PV panel senses overvoltage at its grid connection point, it will disconnect itself from the grid. This will remain disconnected as long as voltage violations persist.

The percentage of PV panels that are shutdown over time are displayed in Fig. 4(a) for both cases. The actual power lost is shown in Fig. 4(b). These two figures clearly show

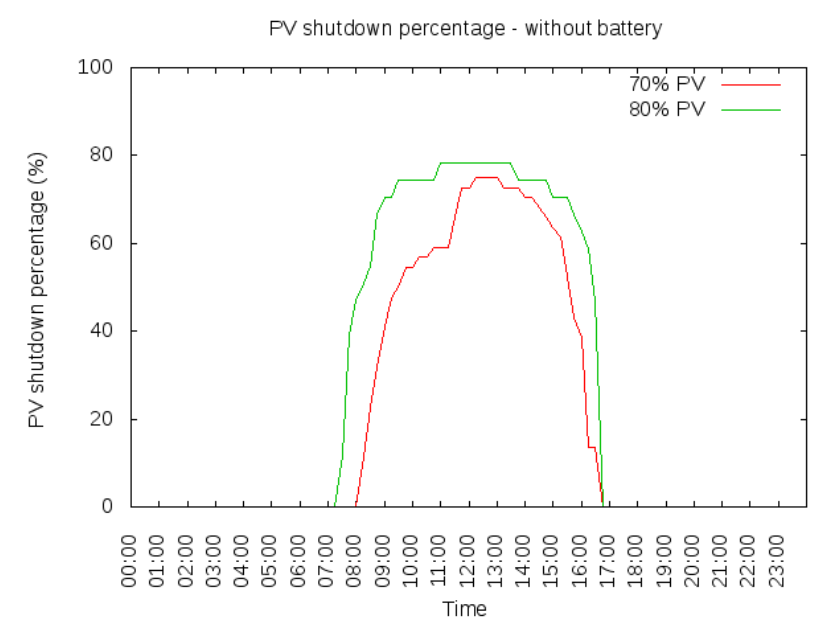

(a) Percentage of PV installations shut off from the grid

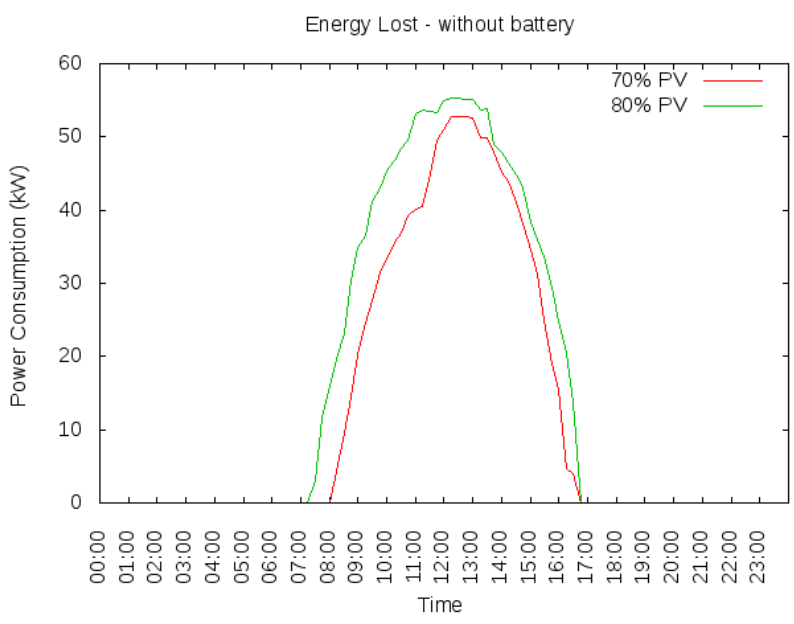

(b) Energy lost due to PV shut-off

Fig. 4. Percentage of PV panels being shut down and the resulting renewable power lost, when no battery is being used.

that more PV panels are being shutdown in the $80 \%$ case and thereby missing out on potentialy green energy. This can be explained by the fact that more panels results in more power being injected in the grid, which results in a overvoltage risk.

To mitigate the problems of unwanted shutdowns of PV panels caused by the voltage instabilities, we introduce a battery in the houses that have a PV panel. The battery we assumed is a rather large model of $9 \mathrm{kWh}$, corresponding to two $4 \mathrm{kWh}$ batteries such as used in [14]. A deterministic algorithm for controlling the charging/discharging of the battery is implemented, charging the battery during the daytime (e.g. when solar power is available) and discharging the battery completely during the night, when no energy is produced by the PV panels: the charge interval is set to 08:00-16:00, while the remaining time the battery is decharged. When discharging the battery, we assume that the released energy is used to power the household appliances. Thus, only if the discharge rate of the battery is higher than the consumption rate of the 


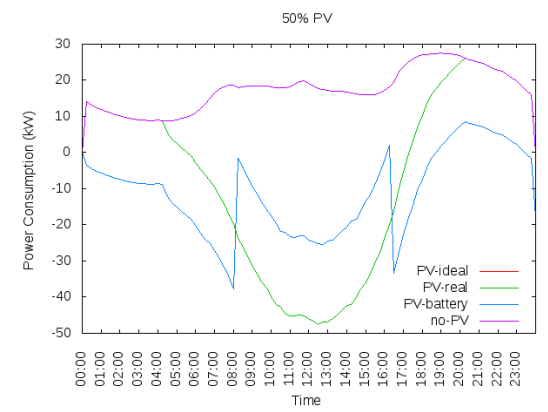

(a) PV penetration $50 \%$

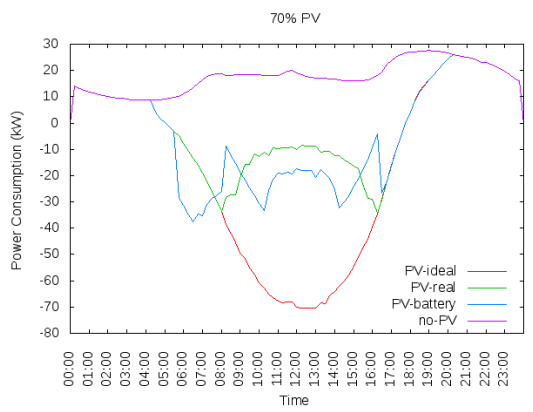

(b) PV penetration $70 \%$

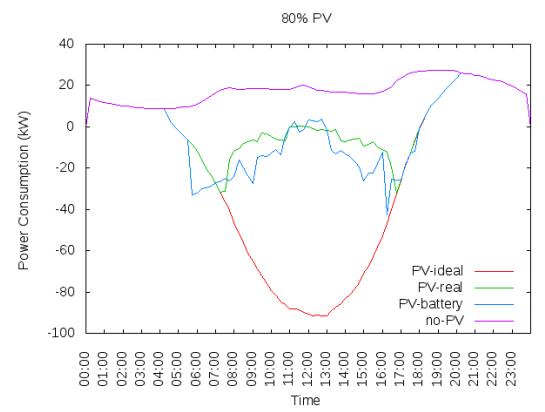

(c) PV penetration $80 \%$

Fig. 3. The net power consumed by the whole of the three feeders comprising the distribution grid spanning 63 houses.

TABLE I

Percentage of PV Panels being ShUtdown \& ENERGy LOST

\begin{tabular}{|l|c|c|}
\hline & Percentage shut down (\%) & Energy lost (kWh) \\
\hline PV-Real 70\% & $34 \%$ & $291 \mathrm{kWh}$ \\
PV-Real: $80 \%$ & $45 \%$ & $482 \mathrm{kWh}$ \\
\hline PV-Battery 70\% & $16 \%$ & $121 \mathrm{kWh}$ \\
PV-Battery $80 \%$ & $28 \%$ & $289 \mathrm{kWh}$ \\
\hline
\end{tabular}

house, excess power is injected into the grid.

Because part of the PV-generated power is now stored in the battery, the impact of PVs on the voltage profile is alleviated. This results in less PV panels having to shutdown due to overvoltage problems, as seen in Fig. 5(a) and Fig. 5(b).

The resulting net flow at the main distribution grid's lowvoltage transformer supplying the three feeders connecting the houses, is now shown in Fig. 3. There, both the lowered production during the day and reduced consumption at night is clearly visible.

Table I gives an overview of the number of PV panels having to shut down for the problematic penetration levels, for both cases (with or without battery). Even the very straightforward (de)charging policy we adopted clearly shows the benefit of providing energy storage. However, we note that there still is an opportunity to optimize the storage capacity and charging policy to further alleviate observed voltage problems (and associated waste of captured solar energy by PV panels).

\section{COnClusion And Future Work}

The primary concern addressed in this paper is the voltage violations caused by the presence of PV panels in a typical residential distribution grid. For a radial grid layout comprising 63 houses, we assessed (i) the net power consumed/produced on the feeders, (ii) the amount of PV panels that would be shut off to avoid voltage violations and (iii) how these problems can be mitigated by providing batteries for storing excess energy. We analyzed this set-up using our simulation tool based on OMNeT++.

Considering an increasing amount of PV installations, we observed that for the case at hand, the grid could sustain 50\% of houses having PV without any problems. However, when PV penetration increases to $70 \%$ (respectively $80 \%$ ), we note that up $34 \%$ (resp. $45 \%$ ) of the PV panels will be shut down

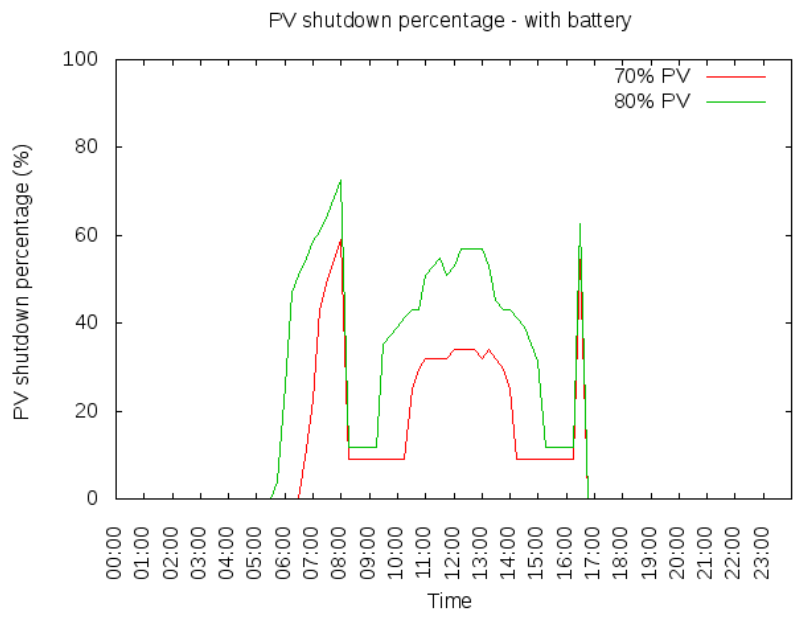

(a) Percentage of PV shut down

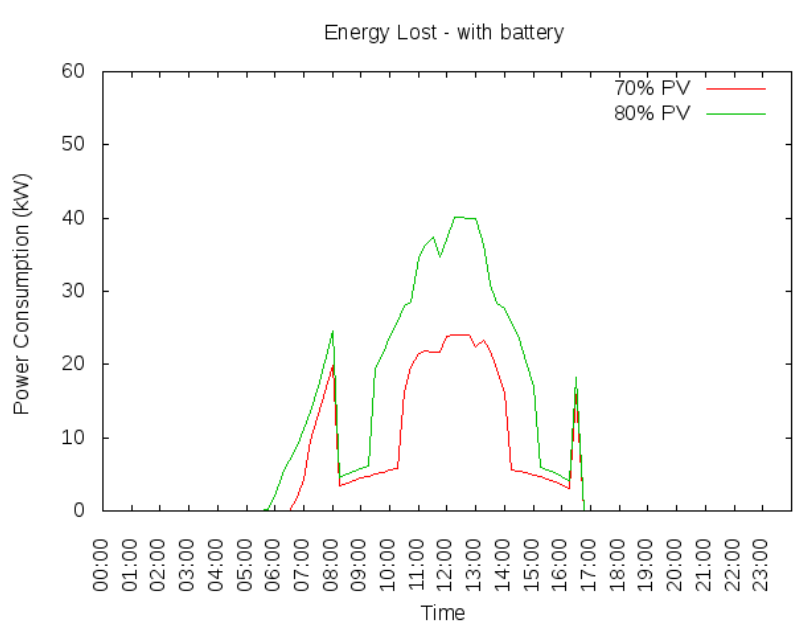

(b) Energy lost due to PV shut-off

Fig. 5. Percentage of PV panels being shut down and the resulting amount of power lost, when using a battery to mitigate voltage problems. 
during daytime. This corresponds $291 \mathrm{kWh}$ (resp. $482 \mathrm{kWh}$ ) of energy that thus is lost. To investigate possible mitigation of this problem, we investigated the effect of adding batteries (9 kAV each) to each of the PV installations. Using a simple, deterministic charge/decharge algorithm set to charge during daytime, and release the energy at nighttime, we saw that we can alleviate many of the unwanted PV shut-offs, and thus recover about half of the otherwise lost energy.

Future work will address (i) investigation of selective/optimized battery installations at possibly a subset of the houses, (ii) the study of ideal battery (de)charging schedules, using e.g. optimisation techniques as in [22], and (iii) online distributed coordination strategies to attain optimal battery (de)charging under uncertain load/production profiles.

\section{ACKNOWLEDGMENT}

Work presented in this paper has been supported by the Flemish Government through the LINEAR project (through IWT) and the project SmartE (an IBBT ICON project). K. Mets would like to thank the Agency for Innovation by Science and Technology in Flanders (IWT) for financial support through his Ph.D. grant. C. Develder is partially supported by the Research Foundation - Flanders (FWO - Vlaanderen) as a post-doctoral fellow.

\section{REFERENCES}

[1] M. Webb, SMART 2020: enabling the low carbon economy in the information age, a report by The Climate Group on behalf of the Global eSustainability Initiative (GeSI), 2008.

[2] E. Coster, J. Myrzik, B. Kruimer, and W. Kling, "Integration issues of distributed generation in distribution grids," Proc. IEEE, vol. 99, no. 1, pp. 28-39, Jan. 2011.

[3] US Department of Energy, "The smart grid: An introduction," 2008.

[4] M. Reza, P. Schavemaker, J. Slootweg, W. Kling, and L. van der Sluis, "Impacts of distributed generation penetration levels on power systems transient stability," in Power Engineering Society General Meeting, 2004. IEEE, june 2004, pp. 2150 -2155 Vol.2.

[5] A. Azmy and I. Erlich, "Impact of distributed generation on the stability of electrical power system," in Power Engineering Society General Meeting, 2005. IEEE, june 2005, pp. 1056 - 1063 Vol. 2.

[6] N. G. A. Hemdan and M. Kurra, "Distributed generation location and capacity effect on voltage stability of distribution networks," in Proc. Annual IEEE Conf. Student Paper, Aalborg, Denmark, 15 Feb. 2008.

[7] M. Chakravorty and D. Das, "Voltage stability analysis of radial distribution networks," International Journal of Electrical Power \& Energy Systems, vol. 23, no. 2, pp. 129 - 135, 2001.
[8] W. Kempton and J. Tomic, "Vehicle-to-grid power implementation: From stabilizing the grid to supporting large-scale renewable energy," Journal of Power Sources, vol. 144, no. 1, pp. 280-294, 2005.

[9] K. Liyanage, A. Yokoyama, Y. Ota, T. Nakajima, and H. Taniguchi, "Impacts of communication delay on the performance of a control scheme to minimize power fluctuations introduced by renewable generation under varying v2g vehicle pool size," in Smart Grid Communications (SmartGridComm), 2010 First IEEE International Conference on, oct. 2010, pp. $85-90$.

[10] E. Furling, M. Piemontesi, P. Prasad, and D. Sukumar, "Advances in energy storage techniques for critical power systems," in The Battcon 2002 proceedings, 2002.

[11] A. Mohd, E. Ortjohann, A. Schmelter, N. Hamsic, and D. Morton, "Challenges in integrating distributed energy storage systems into future smart grid," in Proc. 2008 IEEE Int. Symp. Industrial Electronics, 2008.

[12] F. Geth, J. Tant, E. Haesen, J. Driesen, and R. Belmans, "Integration of energy storage in distribution grids," in Proc. IEEE Power and Energy Society General Meeting, 2010.

[13] P. Vytelingum, T. D. Voice, S. D. Ramchurn, A. Rogers, and N. R Jennings, "Agent-based micro-storage management for the smart grid," in Proc. 9th Int. Conf. Autonomous Agents and Multiagent Sys., ser. AAMAS '10. Richland, SC: International Foundation for Autonomous Agents and Multiagent Systems, 2010, pp. 39-46.

[14] G. Kalogridis, C. Efthymiou, S. Z. Denic, T. A. Lewis, and R. Cepeda, "Privacy for smart meters: Towards undetectable appliance load signatures," in Proc. 1st IEEE Int. Conf. Smart Grid Commun. (SmartGridComm 2010), Gaithersburg, MD, USA, 4-6 Oct. 2010, pp. 85-90.

[15] , "ICT for energy efficiency," EU Commission, Tech. Rep., 2008.

[16] G. Andersson, M. D. Ilić and, V. Madani, and D. Novosel, "Network systems engineering for meeting the energy and environmental dream," Proc. IEEE, vol. 99, no. 1, pp. 7-14, Jan. 2011.

[17] A. Varga and R. Hornig, "An overview of the OMNeT++ simulation environment," in Proc. 1st Int. Conf. Simulation Tools and Techniques for Commun., Networks and Systems (Simutools 2008), Marseilles, France, 3-7 Mar. 2008, pp. 60:1-60:10.

[18] K. Mets, T. Verschueren, C. Develder, T. L. Vandoorn, and L. Vandevelde, "Integrated simulation of power and communication networks for smart grid applications," in Proc. 16th IEEE Int. Workshop Computer Aided Modeling, Analysis and Design of Commun. Links and Netw. (CAMAD 2011), Kyoto, Japan, 10-11 Jun. 2011.

[19] L. Degroote, L. Vandevelde, and R. B., "Fast harmonic simulation model for the analysis of network losses with converter-connecter distributed generation," Electric Power System Research, vol. 80, pp. 1332-1340, Nov. 2010.

[20] VREG, "Flemish regulation entity for the electricity and gas market," Jan. 2010. [Online]. Available: http://www.vreg.be/

[21] PVGIS, "Photovoltaic geographical information system (pvgis)," Jan. 2011. [Online]. Available: http://re.jrc.ec.europa.eu/pvgis/

[22] K. Mets, T. Verschueren, W. Haerick, C. Develder, and F. De Turck, "Optimizing smart energy control strategies for plug-in hybrid electric vehicle charging," in Proc. 1st IFIP/IEEE Int. Workshop on Management of Smart Grids, at 2010 IEEE/IFIP Netw. Operations and Management Symp. (NOMS 2010), Osaka, Japan, 19-23 Apr. 2010, pp. 293-299. 